\title{
Alcoholic versus aqueous chlorhexidine for skin antisepsis: the AVALANCHE trial
}

\author{
Daniel Charles MBBS, Clare F. Heal PhD MBChB, Meth Delpachitra, Michael Wohlfahrt MBBS, \\ Debbie Kimber RN, Julie Sullivan RN, Sheldon Browning MBBS, Sabine Saednia MD, Alexandra Hardy MBBS, \\ Jennifer Banks PhD, Petra Buttner PhD
}

Cite as: CMAJ 2017 August 8;189:E1008-16. doi: 10.1503/cmaj.161460

\begin{abstract}
BACKGROUND: Preoperative skin antisepsis is routine practice. We compared alcoholic chlorhexidine with aqueous chlorhexidine for skin antisepsis to prevent surgical site infection after minor skin excisions in general practice.
\end{abstract}

METHODS: We conducted this prospective, multicentre, randomized controlled trial in 4 private general practices in North Queensland, Australia, from October 2015 to August 2016. Consecutive adult patients presenting for minor skin excisions were randomly assigned to undergo preoperative skin antisepsis with $0.5 \%$ chlorhexidine in $70 \%$ ethanol (intervention) or $0.5 \%$ chlorhexidine aqueous solution (control). Our primary outcome was surgical site infection within 30 days of excision. We also measured the incidence of adverse reactions.

RESULTS: A total of 916 patients were included in the study: 454 underwent antisepsis with alcoholic chlorhexidine and 462 with aqueous chlorhexidine. Of these, 909 completed follow-up. In the intention-to-treat analysis of cases available at follow-up, there was no significant difference in the incidence of surgical site infection between the alcoholic chlorhexidine arm (5.8\%, 95\% confidence interval $[\mathrm{Cl}] 3.6 \%$ to $7.9 \%$ ) and the aqueous chlorhexidine arm (6.8\%,
$95 \% \mathrm{Cl} 4.5 \%$ to $9.1 \%)$. The attributable risk reduction was $0.010(95 \% \mathrm{Cl}-0.021$ to 0.042$)$, the relative risk was 0.85 (95\% $\mathrm{Cl} 0.51$ to 1.41 ), and the number needed to treat to benefit was 100 . Per protocol and sensitivity analyses produced similar results. The incidence of adverse reactions was low, with no difference between groups $(p=0.6)$.

INTERPRETATION: There was no significant difference in efficacy between alcoholic and aqueous chlorhexidine for the prevention of surgical site infection after minor skin excisions in general practice. Trial registration: https://www.anzctr. org.au, no. ACTRN12615001045505 $t$ is routine practice before surgery to cleanse the skin with an antiseptic preparation at the site of surgical incision, a process known as preoperative skin antisepsis. ${ }^{1,2}$ This prevents surgical site infection by removing microorganisms on the skin..$^{1,3,4}$ The consequences of surgical site infection include patient discomfort, functional impairment and increased health care costs. ${ }^{1,3-5}$

The most commonly used preoperative skin antiseptic preparations are povidone iodine and chlorhexidine. Both are available in aqueous and alcoholic preparations, ${ }^{1,3,4}$ and both are effective against a wide range of bacteria, viruses and fungi, although chlorhexidine has more residual antiseptic activity on the skin after application. ${ }^{1,2,4}$ The evidence base guiding appropriate selection of antiseptic agents is poor. A landmark study found that $2 \%$ chlorhexidine in $70 \%$ isopropyl alcohol was superior to aqueous $10 \%$ povidone iodine; however, given that alcohol is known to have important antimicrobial properties, its presence in the chlorhexidine preparation was likely an additional active treatment component. ${ }^{6}$ Recent meta-analyses, including a Cochrane review, concur that it is difficult to make conclusive statements about the relative efficacy of chlorhexidine and povidone iodine ${ }^{1,2,5}$ or about alcoholic and aqueous preparations. ${ }^{1,2}$

Some procedures carry a higher risk for surgical site infection because of body site, pathology and patient factors. Previously identified risk factors include lower leg as the site of the procedure, skin cancer as the reason for excision and diabetes mellitus as a comorbidity. ${ }^{7,8}$

In prior studies of wound infection after minor surgery in general practice in the Mackay region of Australia, our group has shown a surgical site infection rate of about $10 \% .^{7-11}$ The reason for this high rate is unclear, but it may be related to humidity or to patient behaviour in this rural setting. Although this situation is suboptimal, ${ }^{12,13}$ the high infection rate, combined with a high workload for minor surgery in rural general practice, ${ }^{14}$ means that a study of skin antisepsis for the prevention of surgical site infection in our setting is highly feasible. 
Previous research in the Mackay region ${ }^{7,9-11,15}$ and recent interviews (unpublished data) showed that most general practitioners in Mackay use chlorhexidine in preference to povidone iodine, partly because of the perceived "messiness" and skin staining associated with povidone iodine. Therefore, in the current study, we examined the difference between alcoholic and aqueous chlorhexidine, rather than comparing chlorhexidine with povidone iodine.

Alcoholic chlorhexidine has the potential for more pronounced mucosal and ocular irritation ${ }^{16}$ and the danger of operating room fires which, though rare, can have catastrophic consequences. ${ }^{17}$ Several participating clinicians commented that alcoholic chlorhexidine was more likely to remove skin markings made to establish excision margins (unpublished data). We hypothesized that alcoholic chlorhexidine, with independent antibacterial properties, would be superior to aqueous chlorhexidine, and felt that it was important to establish whether a significant difference in efficacy could justify the potential negative effects of the alcoholic preparation.

The aim of our study, the AVALANCHE (Aqueous Versus Alcoholic Antisepsis with Chlorhexidine for Skin Excision) trial, was to compare the effect of alcoholic and aqueous chlorhexidine on the incidence of surgical site infection after minor skin excisions in general practice.

\section{Methods}

\section{Study design and setting}

We conducted this prospective, multicentre assessor-blinded randomized controlled trial (RCT) from October 2015 to August 2016, with reporting in accordance with the CONSORT statement. ${ }^{18}$

We conducted the trial in 3 private general practices in Mackay, Queensland (population 112798), ${ }^{19}$ and 1 private general practice in Mareeba, Queensland (population 7294). ${ }^{20}$

\section{Intervention and control}

For preoperative skin antisepsis, the intervention was $0.5 \%$ chlorhexidine in $70 \%$ ethanol, and the control was $0.5 \%$ chlorhexidine aqueous solution. The $0.5 \%$ concentration aligns with guidelines released by the Queensland Centre for Healthcare Related Infection Surveillance and Prevention. ${ }^{4}$ The $70 \%$ alcoholic concentration is standard for alcoholic preoperative skin preparations. ${ }^{2}$ We purchased the antiseptic solutions from an independent supplier (SSS Australia).

\section{Recruitment and participant eligibility}

Consecutive adult patients presenting for "minor skin excision" (i.e., excision of benign or malignant skin lesions under local anesthetic, performed in general practice) were invited to participate. Practice nurses were responsible for recruitment and treatment assignment. Nurses provided an information sheet to each potential participant before requesting written informed consent.

We excluded patients who were already taking antibiotics. Other exclusion criteria were excision of sebaceous cyst and allergy to alcohol or chlorhexidine. Additional methodologic details are outlined in the study protocol. ${ }^{21}$
We did not plan to exclude periocular excisions; however, during the first week of data collection, 1 patient experienced ocular irritation from an alcoholic solution, and patients with this type of lesion were excluded thereafter. We reported this event as an adverse reaction.

\section{Randomization and blinding}

Randomization was at the level of the patient. We used computer-generated random numbers to create permuted blocks of 50 with a 1:1 ratio. Group assignments were concealed in numbered, sealed, opaque envelopes, which were opened after patients provided consent to participate. The researchers responsible for randomization had no role in participant enrolment, assignment or treatment.

Some features of the alcoholic preparation, such as smell, were identifiable, so blinding of personnel and patients was not feasible. However, the outcome assessor (the treating doctor) was blinded to treatment assignment. In addition, to improve the specificity (but not the sensitivity) of diagnosis of surgical site infection, a single blinded outcome assessor photographed and assessed infected wounds.

\section{Surgical and wound care protocols}

In consultation with the participating clinicians, we developed a protocol modelled upon international guidelines and similar trials. ${ }^{4,7,9,10,15,22}$ The antiseptic solution was applied using sterile forceps and gauze over an area $1 \mathrm{~cm}$ beyond the surgical field. Drapes, gloves, sutures, local anesthetic and dressings were the same in the 2 study arms. Written postoperative wound care instructions were used. In addition, the clinicians used a diathermy protocol to minimize the risk of fires. . $^{417,23}$

Surgical site infection was determined in accordance with a modified version of the Centers for Disease Control and Prevention (CDC) definition. ${ }^{22}$ The infection was required to occur within 30 days of the excision and to involve only skin or subcutaneous tissue. Additionally, at least 1 of the following had to have occurred: purulent discharge with or without laboratory confirmation from the superficial excision; at least 1 of pain or tenderness, localized swelling, redness or heat; or diagnosis of superficial infection by the physician. Stitch abscess was not included in this definition. ${ }^{9,15,22}$

\section{Outcome measures}

The primary outcome measure was the incidence of surgical site infection within 30 days after the excision. Wounds were assessed when the patients presented for removal of sutures, for evaluation of signs or symptoms of surgical site infection or for any other reason (opportunistically).

The secondary outcome measures were adverse reactions to the preoperative skin antiseptic agent (manifesting as any one of anaphylaxis, skin irritation, contact dermatitis or rash), the results of microbiology testing of infected wounds with purulent discharge and any patterns of antibiotic resistance. Ocular irritation was classified as an adverse reaction.

Participating clinicians were given standardized education about the diagnosis of surgical site infection. The clinicians pre- 
scribed antibiotics for such infections if clinically indicated and, for wounds with a purulent discharge, obtained samples for microscopy, culture and sensitivity (as per standard practice).

\section{Data collection}

Staff at each practice location collected baseline data for patient, excision and lesion characteristics, based on risk factors for surgical site infection identified in previous trials. ${ }^{7-9,15}$ Excision location was recorded on a body site map. Clinicians at each practice site examined patient records to complete missing data.

\section{Statistical analysis}

For determination of sample size, we predicted a baseline infection rate of $10 \%$, on the basis of pooled data from 3 previous studies of surgical site infection in the Mackay region, ${ }^{7,9,10}$ which used aqueous chlorhexidine. We contacted participating clinicians individually to seek input on the level of reduction in the incidence of surgical site infection that would be considered clinically significant; these clinicians judged that an absolute reduction in incidence of $5 \%$ (i.e., to $5 \%$ ) with alcoholic chlorhexidine would be clinically significant. To detect a difference of this magnitude with statistical confidence, power in excess of $80 \%$ and a 2-tailed significance level of 0.05 , a total of 435 patients was required in each of the intervention and control groups. To counter potential attrition, and given dropout rates less than $5 \%$ in previous studies, ${ }^{7,9,10,15}$ the target sample size was increased to 920 .

We compared the intervention and control groups in terms of baseline characteristics of patients, lesions and excisions.

We compared the incidence of surgical site infection (the primary outcome variable) in the intervention and control groups using the Fisher exact test and 95\% confidence intervals (Cls). In addition, we calculated the attributable risk reduction, the relative risk, the number needed to treat (NNT) and the number neeed to harm ( $\mathrm{NNH})^{24}$ with $95 \%$ Cls.

The primary analysis was an intention-to-treat analysis based on cases available at follow-up, including all participants who underwent randomization, with the individual person as the unit of analysis. We also conducted a per protocol analysis, which excluded participants with protocol violations, and a sensitivity analysis for patients lost to follow-up. In addition, we compared the relative incidence of adverse outcomes between the intervention and control groups using the Fisher exact test. All reported $p$ values were 2 tailed, and for each analysis $p$ less than 0.05 was considered statistically significant.

Data were analyzed with SPSS Statistics version 24 (IBM).

\section{Ethics approval}

This trial received ethics approval from the James Cook University Human Research Ethics Committee (H6065) and was registered with the Australian and New Zealand Clinical Trials Registry (ACTRN12615001045505).

\section{Results}

Over the study period, 1041 patients were assessed for eligibility and met the inclusion criteria, of whom 125 were excluded (Figure 1).
Of the remaining 916 patients, 454 were assigned to undergo skin antisepsis with alcoholic chlorhexidine (intervention) and 462 with aqueous chlorhexidine (control). Seven of the patients $(0.8 \%)$ were lost to follow-up, which left 909 patients who qualified for the intention-to-treat analysis of available cases. In 16 $(1.7 \%)$ of the 916 cases, the wound protocol was violated. In total, 14 general practitioners at 4 general practices performed between 1 and 249 procedures that contributed to the trial.

The mean age (standard deviation [SD]) of excluded patients was significantly higher than the age of those who underwent randomization (69.2 [SD 14.7) yr v. 64.9 [SD 14.0] yr, $p=0.002$ ). There was no difference in sex ratio (proportions of men: $72 / 125$ [57.6\%] v. 517/916 [56.4\%], $p=0.8)$.

At baseline, there were no large differences between the intervention and control groups in terms of patient, lesion or excision characteristics (Table 1).

\section{Incidence of surgical site infection}

In the intention-to-treat analysis of cases available at followup, surgical site infection occurred in $57(6.3 \%, 95 \% \mathrm{Cl} 4.7 \%$ to $7.9 \%)$ of the 909 patients. There was no significant difference between the alcoholic and aqueous chlorhexidine arms $(5.8 \%$ [26/451], $95 \% \mathrm{Cl} 3.6 \%$ to $7.9 \%$ v. $6.8 \%$ [31/458], $95 \% \mathrm{Cl} 4.5 \%$ to $9.1 \%)$. The attributable risk reduction was $0.010(95 \% \mathrm{Cl}-0.021$ to 0.042$)$, relative risk was $0.85(95 \% \mathrm{Cl} 0.51$ to 1.41$)$, and the NNT was 100.

The per protocol analysis also showed no significant difference between the groups (attributable risk reduction 0.012, 95\% $\mathrm{Cl}-0.020$ to 0.044 ; NNT 84). In the sensitivity analysis, there was no significant difference whether all 7 patients lost to follow-up were assumed to have infection or no infection (Table 2).

About one-third (20/57 [35.1\%]) of the infections were photographed and rated by an independent outcome assessor, with agreement for all 20 of these cases.

\section{Adverse reactions}

One episode of ocular irritation was reported in the alcoholic chlorhexidine arm, and 3 episodes of contact dermatitis were reported in the aqueous chlorhexidine arm (combined adverse effects, $p=0.6$; contact dermatitis, $p=0.2$ ). No other adverse reactions attributable to the antiseptic preparations were reported.

\section{Microbiologic testing of infected wounds}

The results of microbiologic testing were reported for $7(0.8 \%)$ of the patients ( 5 in the intervention group, 2 in the control group). All samples grew Staphylococcus aureus, and 1 sample from the alcoholic chlorhexidine arm additionally grew Pseudomonas aeruginosa. Sensitivity testing for 2 of the samples showed pansensitive S. aureus.

\section{Interpretation}

No statistically or clinically significant differences were found between alcoholic and aqueous chlorhexidine for the prevention of surgical site infection after minor skin excisions in general practice. 
These findings are consistent with previous studies of both clean surgery (i.e., gastrointestinal, genitourinary or respiratory tract not entered) and other classifications of surgical contamination (Table 3), which have mostly shown a trend favouring alcohol-based skin antisepsis, but no significant differences. ${ }^{6,25-36}$

In our study, there was a low incidence of contact dermatitis, and no significant difference between groups, consistent with similarly low incidences ( $0 \%$ to $0.8 \%$ ) reported in the literature. .,29,34 $^{2}$

Staphylococcus aureus was the primary organism cultured from wound swabs, again consistent with previous studies. ${ }^{30,31}$

\section{Limitations}

In this trial, we addressed a question that is relevant to clinicians and readily translated into clinical practice. Few large RCTs are conducted in primary care because of practical and funding difficulties. ${ }^{38,39}$ However, it is important that clinical guidelines used in general practice be informed by primary care evidence, so that they will be directly applicable to patients who present to the general practitioner. ${ }^{40}$

The study had some limitations. The baseline infection rate was lower than we predicted on the basis of previous studies. ${ }^{7-10}$ Our sample size calculation was based on a $5 \%$ absolute reduction in surgical site infection (from a $10 \%$ baseline rate) being clinically relevant. It is possible that general practitioners might have reconsidered the margin for superiority if the baseline rate were $7 \%$. The observed difference might be considered clinically relevant to a clinician working in a setting with a lower baseline infection rate. ${ }^{41-43}$

Although the gold standard, the diagnosis of surgical site infection as per CDC criteria, is subjective and prone to inter- and intra-observer variability, ${ }^{44}$ we could not blind personnel to the intervention. Furthermore, it was not financially or practically

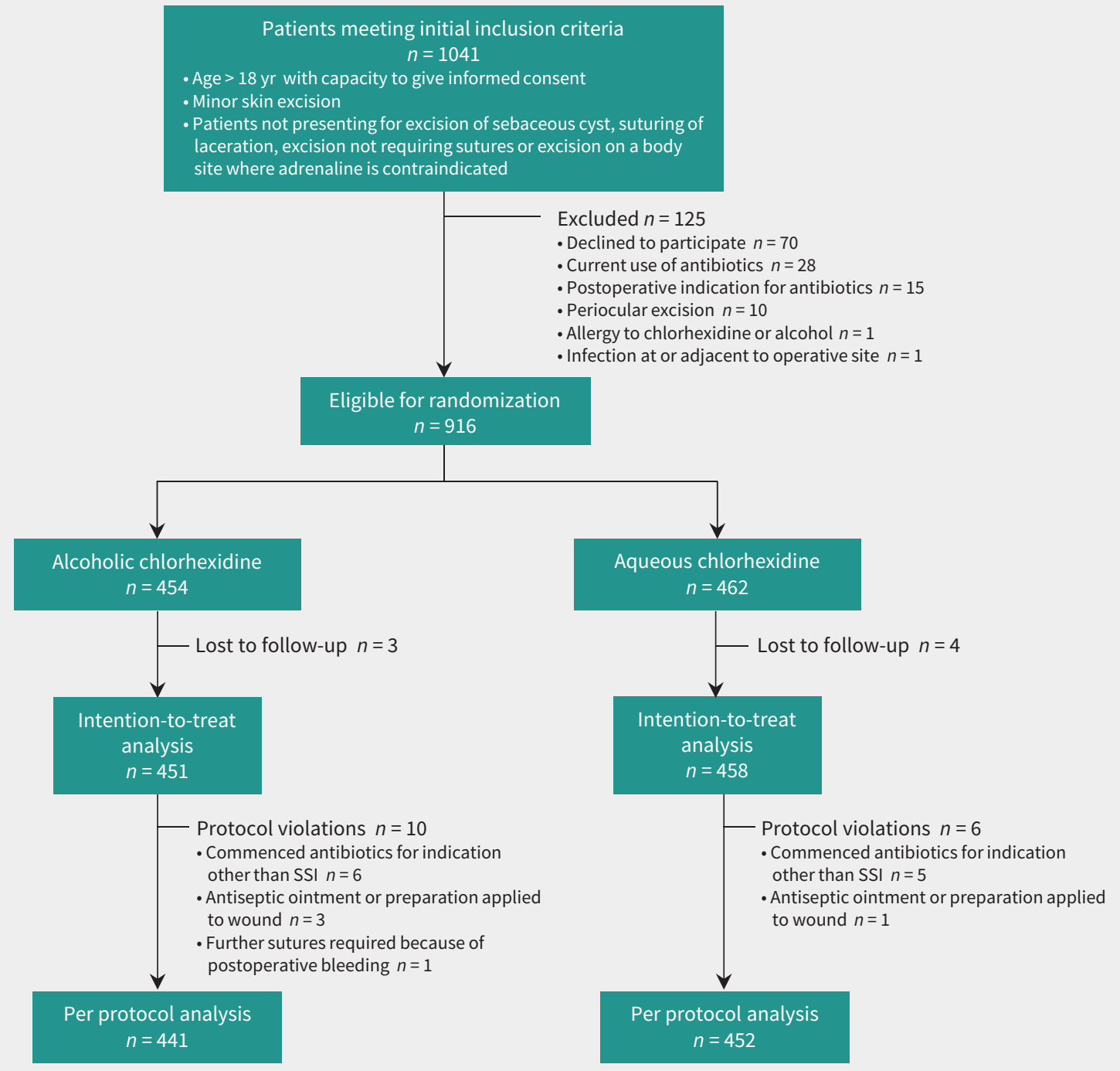

Figure 1: Flow chart of enrolment, assignment, follow-up and analysis of study participants. ${ }^{21} \mathrm{SSI}=$ surgical site infection. 
Table 1: Baseline characteristics of patients, lesions and excisions, by treatment group

Group; no. (\%) of patients*

Alcoholic chlorhexidine

(intervention)

$n=454$

\section{Characteristic}

\section{Patient}

Age, yr, mean \pm SD (range)

Sex, male

Smoking status

Current smoker

Ex-smoker

Never smoked

Comorbid conditions

Any†

Diabetes mellitus

Ischemic heart disease

COPD

Medications

Taking a medication

Oral anticoagulant agents

Oral antiplatelet agents

Oral or inhaled steroids or other immunosuppressant

\section{Lesion}

Body site

Head and neck

Trunk

Upper limb

Lower limb: below knee

Lower limb: above knee

Histologic findings

Malignant melanoma

Basal cell carcinoma or squamous cell carcinoma

Cancerous precursor§

Other lesion

\section{Excision}

Type of excision

Simple

Two-layer or flap

Suture size

Thick $(2 / 0$ or $3 / 0)$

Thin $(4 / 0,5 / 0$ or $6 / 0)$

Multiple

Length of excision, $\mathrm{mm}$, median (range)

Time to removal of sutures, $d$, mean \pm SD (range)
$65.1 \pm 14.2(21-91)$

$266(58.6)$

$33(7.3)$

$128(28.2)$

$293(64.5)$

$99(21.8)$

$105(22.7)$

$44(9.7)$

$53(11.5)$

$43(9.5)$

$53(11.5)$

$16(3.5)$

$10(2.2)$

$131(28.9)$

$128(27.7)$

65 (14.3)

75 (16.2)

42 (9.3)

$35(7.6)$

$39(8.6)$

$30(6.5)$

154 (33.9)

84 (18.5)

174 (37.7)

$114(25.1)$

$81(17.5)$

$127(27.5)$

80 (17.6)

64 (13.9)

$22(4.8)$

$16(3.5)$

$11(2.4)$

9 (1.9)

257 (56.6)

254 (55.0)

69 (15.2)

77 (16.7)

$117(25.8)$

$122(26.4)$

443 (97.6)

453 (98.1)

$11(2.4)$

$9(1.9)$

277 (61.0)

269 (58.2)

$116(25.6)$

$130(28.1)$

61 (13.4)

63 (13.6)

$25.0(3-100)$

$25.0(3-100)$

$9.69 \pm 2.95(3-19)$

Note: $\mathrm{COPD}=$ chronic obstructive pulmonary disease, $\mathrm{SD}=$ standard deviation

*Except where indicated otherwise.

tComorbid conditions were diabetes mellitus, peripheral vascular disease, ischemic heart disease, cancer and inflammatory skin disease.

$\ddagger$ Medications were steroids (oral or inhaled), anticoagulants, antiplatelet agents, opioids (oral or cutaneous) and other immunosuppressive medications.

$\S$ Cancerous precursors were actinic keratoses and dysplastic nevi. 
feasible for a single outcome assessor to assess each wound at the 4 geographically dispersed practices. ${ }^{21}$ An independent outcome assessor rated photographs of some infections to increase the specificity (but not the sensitivity) of diagnosis; however, only $37.5 \%$ of infections were photographed. Only 7 infected wounds were sampled for bacterial culture, and for only 2 of these was antibiotic sensitivity reported; therefore, data were insufficient to draw conclusions about antibiotic resistance.

Many factors influence surgical site infection, and some potentially important variables were inadequately recorded, such as the patient's occupation and skin integrity. Also, medical history is subject to recall bias. Other potential confounders, such as the doctor's surgical skill, could not be measured or recorded.

We did not adjust for the possible effect of clustering by general practitioner or by practice; however, in previous similar trials, ${ }^{7,9,10,15}$ these factors did not produce a large design effect. Had we adjusted for a potential clustering effect, the estimated variances would likely have been larger, and the nonsignificant results would have been emphasized.
The Cochrane definition of intention to treat is the analysis of all patients who underwent randomization, regardless of whether the protocol was violated or the patients were lost to follow-up. ${ }^{45}$ If data were imputed, on the basis of baseline infection rates, it is likely that the 7 patients lost to follow-up would be classified as being without infection. Our intention-to-treat analysis is presented as an available-case analysis (including patients with protocol violations but not those lost to follow-up). Our sensitivity analysis is, in effect, a data imputation, in accordance with the Cochrane definition.

The populations of Mackay and Mareeba differ slightly from the general Australian population. ${ }^{19,20}$ The incidence of surgical site infection in our tropical setting was higher than that for cohorts in other areas, but the relative efficacy of aqueous and alcoholic chlorhexidine is unlikely to change with climate. Our results are consistent with those of trials comparing alcoholic with aqueous preparations in clean general surgery, ${ }^{25,30,31,33,35}$ and the results may be generalizable to this setting, as well as to other simple general practice procedures, such as insertion of a contraceptive device.

Table 2: Incidence of surgical site infection in intervention and control groups

\begin{tabular}{|c|c|c|c|c|}
\hline \multirow[b]{2}{*}{ Analysis } & \multicolumn{2}{|c|}{ Group; no. or \% } & \multirow[b]{2}{*}{ ARR $(95 \% \mathrm{Cl})$ and $\mathrm{NNT}^{28 *}$} & \multirow[b]{2}{*}{ RR $(95 \% \mathrm{Cl})$} \\
\hline & $\begin{array}{l}\text { Alcoholic chlorhexidine } \\
\text { (intervention) }\end{array}$ & $\begin{array}{l}\text { Aqueous chlohexidine } \\
\text { (control) }\end{array}$ & & \\
\hline \multicolumn{5}{|l|}{ Intention-to-treat analysis } \\
\hline No. of participants & 451 & 458 & & \\
\hline No. of infections & 26 & 31 & & \\
\hline Incidence of infection, \% & 5.8 & 6.8 & $\begin{array}{c}\quad 0.010(-0.021 \text { to } 0.042) \\
\text { NNT } 100 \\
\text { (NNH } 48 \text { to infinity to NNT 24) }\end{array}$ & $0.85(0.51$ to 1.41$)$ \\
\hline \multicolumn{5}{|l|}{ Per protocol analysis } \\
\hline No. of participants & 441 & 452 & & \\
\hline No. of infections & 25 & 31 & & \\
\hline Incidence of Infection, \% & 5.7 & 6.9 & $\begin{array}{c}0.012(-0.020 \text { to } 0.044) \\
\text { NNT } 84 \\
\text { (NNH } 50 \text { to infinity to NNT 23) }\end{array}$ & 0.83 (0.50 to 1.38$)$ \\
\hline \multicolumn{5}{|l|}{ Sensitivity analyses } \\
\hline \multicolumn{5}{|c|}{ Lost to follow-up assumed without infection } \\
\hline No. of participants & 454 & 462 & & \\
\hline No. of infections & 26 & 31 & & \\
\hline Incidence of infection, \% & 5.7 & 6.7 & $\begin{array}{l}0.0098(-0.021 \text { to } 0.041) \\
\text { NNT } 102 \\
\text { (NNH } 48 \text { to infinity to NNT 24) }\end{array}$ & $0.85(0.52$ to 1.41$)$ \\
\hline \multicolumn{5}{|c|}{ Lost to follow-up assumed with infection } \\
\hline No. of participants & 454 & 462 & & \\
\hline No. of infections & 29 & 35 & & \\
\hline Incidence of infection, \% & 6.4 & 7.6 & $\begin{array}{c}\quad 0.012(-0.021 \text { to } 0.045) \\
\text { NNT } 84 \\
\text { (NNH } 47 \text { to infinity to NNT 22) }\end{array}$ & 0.84 (0.52 to 1.36$)$ \\
\hline
\end{tabular}


Table 3: Previous studies comparing alcoholic and aqueous preparations for prevention of surgical site infection

\section{Study, date \\ and location}

Abreu et al. ${ }^{36}$

(2014), Uruguay

Bibbo et al. ${ }^{26}$

(2005), USA

Brown et al. ${ }^{27}$

(1984), USA $\dagger$

Darouiche et al. ${ }^{6}(2010)$, USA

Gilliam et al. ${ }^{28}$

(1990), USA

Howard ${ }^{33}$

(1991), USA

Meier et al. ${ }^{30}$

(2001), Nigeriał

Paocharoen et

al. ${ }^{29}$ (2009),

Thailand $\dagger$

Paocharoen et al. ${ }^{29}$ (2009), clean surgeries only, Thailand $\ddagger$

Roberts et al. ${ }^{35}$ (1995), USA

Saltzman et al. ${ }^{32}$ (2009), USA

Segal and Anderson ${ }^{25}$ (2002), USA

Sistla et al. ${ }^{31}$

(2010), Indiaf

Srinivas et al. ${ }^{34}$ (2015), India

\section{Study arm; incidence of} infection, $n / N(\%)$

56 participants, clean contaminated

Intervention: $0.5 \%$ alcoholic $\mathrm{CHG}$

Control: $0.5 \%$ aqueous PVI

127 participants, clean

Intervention: $4 \% \mathrm{CHG}$ scrub $+70 \%$ alcohol paint

Control: $7.5 \%$ aqueous $\mathrm{PVI}$ scrub $+10 \%$ aqueous $\mathrm{PVI}$ paint

737 participants, clean, clean contaminated +

contaminated

Intervention: $0.5 \%$ CHG in $70 \%$ alcohol spray

Control: $0.7 \%$ aqueous PVI scrub + aqueous PVI paint

849 participants, clean contaminated

Intervention: $2 \%$ CHG in $70 \%$ alcohol scrub

Control: $10 \%$ aqueous PVI scrub + paint

60 participants, clean

Intervention: $0.7 \%$ iodophor in $74 \%$ alcohol paint

Control: Aqueous iodophor scrub + paint

159 participants, clean

Intervention: alcoholic iodophor

Control: aqueous iodophor scrub + paint

200 participants, clean

Intervention: bath soap scrub + methylated spirit paint

Control: aqueous PVI scrub + paint

500 participants, clean, clean contaminated +

contaminated

Intervention: 4\% CHG in 70\% alcohol scrub + paint

Control: aqueous PVI scrub + paint

183 participants, clean

Intervention: 4\% CHG in 70\% alcohol scrub + paint

Control: aqueous PVI scrub + paint

Alcoholic Aqueous

RR

$6 / 32$

$6 / 32$
$(18.8)$

$4 / 24$

$0 / 60$

(16.7)

$0 / 60$
$(0.0)$

$0 / 67$

(0.0)

$23 / 378$

29/359

(8.1)

0.75

(0.44 to 1.28$)$

(6.1)

$(95 \% \mathrm{Cl})$

ARR, \%

1.13

$+2.2$

$p$ value

$\begin{array}{ccccc}\begin{array}{c}39 / 409 \\ (9.5)\end{array} & \begin{array}{c}71 / 440 \\ (16.1)\end{array} & \begin{array}{c}0.59 \\ (0.41 \text { to } 0.85)\end{array} & -6.6 & 0.004 \\ & & & \\ 0 / 30 & 0 / 30 & \text { NA } & 0.0 & \text { NA } \\ (0.0) & (0.0) & & & \\ & & & & \\ 2 / 84 & 2 / 75 & 0.89 & -0.3 & \text { NS } \\ (2.4) & (2.7) & (0.13 \text { to } 6.18) & & \\ & & & & \\ 5 / 98 & 6 / 102 & 0.87 & -0.8 & \text { NS } \\ (5.1) & (5.9) & (0.27 \text { to } 2.75) & & \\ & & & & \\ 5 / 250 & 8 / 250 & 0.63 & -1.2 & \text { NS } \\ (2.0) & (3.2) & (0.21 \text { to } 1.88) & & \end{array}$

\section{2/96}

$5 / 87$

(5.7)

0.36

$-3.6$

NS

200 participants, clean

Intervention: alcoholic iodophor

Control: aqueous iodophor scrub + paint

150 participants, clean

$10 / 104$

(9.6)

$9 / 96$
$(9.4)$

1.03

$+0.2$

NS

Int 1: 0/50 (0.0)

$0 / 50$

Int 2: 0/50 (0.0)

(0)

Intervention 2: $0.7 \%$ iodophor in $74 \%$ alcohol

Control: $0.75 \% \mathrm{PVI}$ scrub + 1\% PVI paint

209 participants, clean

Intervention 1: alcoholic iodophor

Intervention 2: alcoholic iodophor + incise drape§

Control 1: $10 \%$ aqueous PVI paint

Control 2: $7.5 \%$ aqueous PVI scrub $+10 \%$ aqueous PVI paint

556 participants, clean

Intervention: $2.5 \%$ CHG in 70\% alcohol paint

Control: $10 \%$ aqueous PVI paint

Int $1: 1 / 50$
$(2.0)$

Control 1: $7 / 56$
(12.5)
Control 2: $7 / 52$

0.16

0.15

$-10.5$

NS

(13.5)

$\begin{array}{cc}14 / 200 & 19 / 200 \\ (7.0) & (9.5) \\ & \\ 17 / 158 & 33 / 184 \\ (10.8) & (17.9)\end{array}$

0.74

0.60

$-7.1$

NS

Note: $\mathrm{ARR}=$ absolute risk reduction, $\mathrm{CHG}=$ chlorhexidine, $\mathrm{Cl}=$ confidence interval, $\mathrm{NA}=$ not available, $\mathrm{NS}=$ no statistically significant difference between intervention and control ( $p$ value not reported), $\mathrm{PVI}=$ povidone iodine, $\mathrm{RR}=$ relative risk.

* "Clean" = noncontaminated wound; "clean contaminated" = operative wound in respiratory, alimentary or genitourinary tract, or minor break in aseptic technique; "contaminated" = open, fresh, accidental wound, acute nonpurulent inflammation, gross spillage from gastrointestinal tract or major break in aseptic technique. ${ }^{37}$

tData supplemented by analyses performed in systematic review of literature by Maiwald and colleagues. ${ }^{2}$

$\ddagger$ Data supplemented by analyses performed in systematic review of literature by Dumville and colleagues. ${ }^{1}$

$\S D$ ata from this group were excluded from analysis because of use of incise drape as an additional intervention. 


\section{Conclusion}

The AVALANCHE trial showed no clinically relevant or statistically significant difference between alcoholic and aqueous chlorhexidine for skin antisepsis for the prevention of surgical site infection after clean minor surgery in general practice.

\section{References}

1. Dumville JC, McFarlane E, Edwards P, et al. Preoperative skin antiseptics for preventing surgical wound infections after clean surgery. Cochrane Database Syst Rev 2015;(4):CD003949.

2. Maiwald M, Chan ES. The forgotten role of alcohol: a systematic review and meta-analysis of the clinical efficacy and perceived role of chlorhexidine in skin antisepsis. PLoS One 2012;7:e44277.

3. Lee I, Agarwal RK, Lee BY, et al. Systematic review and cost analysis comparing use of chlorhexidine with use of iodine for preoperative skin antisepsis to prevent surgical site infection. Infect Control Hosp Epidemiol 2010;31:1219-29.

4. Surgical skin disinfection guideline. Version 3.0. Queensland (Australia): Queensland Government, Department of Health; 2015. Available: https://www.health.qld.gov.au /_data/assets/pdf_file/0020/444422/skin-disinfection.pdf (accessed 2017 June 28).

5. Kamel C, McGahan L, Mierwinski-Urban M, et al. Preoperative skin antiseptic preparations and application techniques for preventing surgical site infections: a systematic review of the clinical evidence and guidelines. Ottawa: Canadian Agency for Drugs and Technologies in Health; 2011.

6. Darouiche RO, Wall MJ Jr, Itani KM, et al. Chlorhexidine-alcohol versus povidone-iodine for surgical-site antisepsis. N Engl J Med 2010;362:18-26.

7. Heal C, Buettner P, Raasch B, et al. Can sutures get wet? Prospective randomised controlled trial of wound management in general practice. BMJ 2006;332:1053-6.

8. Heal CF, Buettner PG, Drobetz H. Risk factors for surgical site infection after dermatological surgery. Int J Dermatol 2012;51:796-803.

9. Heal C, Sriharan S, Buttner PG, et al. Comparing non-sterile to sterile gloves for minor surgery: a prospective randomised controlled non-inferiority trial. Med $\mathrm{J}$ Aust 2015;202:27-31.

10. Heal CF, Buettner PG, Cruickshank R, et al. Does single application of topical chloramphenicol to high risk sutured wounds reduce incidence of wound infection after minor surgery? Prospective randomised placebo controlled double blind trial. BMJ 2009;338:a2812.

11. Heal C, Buettner P, Browning S. Risk factors for wound infection after minor surgery in general practice. Med J Aust 2006;185:255-8.

12. Culver DH, Horan TC, Gaynes RP, et al. Surgical wound infection rates by wound class, operative procedure, and patient risk index. National Nosocomial Infections Surveillance System. Am J Med 1991;91:152S-157S.

13. Platt R. Antibiotic prophylaxis in clean surgery: Does it work? Should it be used if it does? New Horiz 1998;6(Suppl):S53-7.

14. Askew DA, Wilkinson D, Schluter PJ, et al. Skin cancer surgery in Australia 20012005: the changing role of the general practitioner. Med J Aust 2007;187:210-4.

15. Smith SC, Heal CF, Buttner PG. Prevention of surgical site infection in lower limb skin lesion excisions with single dose oral antibiotic prophylaxis: a prospective randomised placebo-controlled double-blind trial. BMJ Open 2014;4:e005270.

16. Pfizer Global Environment, Health, and Safety Operations, Product Stewardship Hazard Communication. Material safety data sheet: chlorhexidine in alcohol 70\%. New York: Pfizer Inc.; 2012.

17. Tooher R, Maddern GJ, Simpson J. Surgical fires and alcohol-based skin preparations. ANZ J Surg 2004;74:382-5.

18. Schulz KF, Altman DG, Moher D; CONSORT Group. CONSORT 2010 statement: updated guidelines for reporting parallel group randomised trials. BMJ 2010; 340:c332.
19. 2011 census QuickStats: Mackay. Canberra (Australia): Australian Bureau of Statistics; 2013. Available: www.abs.gov.au/websitedbs/censushome.nsf/home/ quickstats (accessed 2016 July 24).

20. 2011 census Quickstats: Mareeba. Canberra (Australia): Australian Bureau of Statistics; 2013. Available: www.abs.gov.au/websitedbs/censushome.nsf/ home/quickstats (accessed 2016 July 24).

21. Heal CF, Charles D, Hardy A, et al. Protocol for a randomised controlled trial comparing aqueous with alcoholic chlorhexidine antisepsis for the prevention of superficial surgical site infection after minor surgery in general practice: the AVALANCHE trial. BMJ Open 2016;6:e011604.

22. Mangram AJ, Horan TC, Pearson ML, et al. Guideline for prevention of surgical site infection, 1999. Hospital Infection Control Practices Advisory Committee. Infect Control Hosp Epidemiol 1999;20:250-78, quiz 279-80.

23. Association of periOperative Registered Nurses. AORN guidance statement: fire prevention in the operating room [published erratum in AORN J 2005;82:16]. AORN J 2005;81:1067-75.

24. Altman DG. Confidence intervals for the number needed to treat. $B M J$ 1998;317:1309-12.

25. Segal CG, Anderson JJ. Preoperative skin preparation of cardiac patients. AORN J 2002;76:821-8.

26. Bibbo C, Patel DV, Gehrmann RM, et al. Chlorhexidine provides superior skin decontamination in foot and ankle surgery: a prospective randomized study. Clin Orthop Relat Res 2005;438:204-8.

27. Brown TR, Ehrlich CE, Stehman FB, et al. A clinical evaluation of chlorhexidine gluconate spray as compared with iodophor scrub for preoperative skin preparation. Surg Gynecol Obstet 1984;158:363-6.

28. Gilliam DL, Nelson CL. Comparison of a one-step iodophor skin preparation versus traditional preparation in total joint surgery. Clin Orthop Relat Res 1990;250:258-60.

29. Paocharoen V, Mingmalairak C, Apisarnthanarak A. Comparison of surgical wound infection after preoperative skin preparation with $4 \%$ chlorhexidine [correction of chlohexidine] and povidone iodine: a prospective randomized trial. J Med Assoc Thai 2009;92:898-902.

30. Meier DE, Nkor SK, Aasa D, et al. Prospective randomized comparison of two preoperative skin preparation techniques in a developing world country. World J Surg 2001;25:441-3.

31. Sistla SC, Prabhu G, Sistla S, et al. Minimizing wound contamination in a 'clean' surgery: comparison of chlorhexidine-ethanol and povidone-iodine. Chemotherapy 2010;56:261-7.

32. Saltzman MD, Nuber GW, Gryzlo SM, et al. Efficacy of surgical preparation solutions in shoulder surgery. J Bone Joint Surg Am 2009;91:1949-53.

33. Howard R. Comparison of a 10-minute aqueous iodophor and 2-minute waterinsoluble iodophor in alcohol preoperative skin preparation. Complicat Surg 1991;10:43-5.

34. Srinivas A, Kaman L, Raj P, et al. Comparison of the efficacy of chlorhexidine gluconate versus povidone iodine as preoperative skin preparation for the prevention of surgical site infections in clean-contaminated upper abdominal surgeries. Surg Today 2015;45:1378-84.

35. Roberts A, Wilcox K, Devineni R, et al. Skin preparation in CABG surgery: a prospective randomised trial. Complicat Surg 1995;1:724, 741-4, 747.

36. Abreu D, Campos E, Seija V, et al. Surgical site infection in surgery for benign prostatic hyperplasia: comparison of two skin antiseptics and risk factors. Surg Infect (Larchmt) 2014;15:763-7.

37. Heal CF, Banks JL, Lepper PD, et al. Topical antibiotics for preventing surgical site infection in wounds healing by primary intention. Cochrane Database Syst Rev 2016;11:CD011426. 
38. Mendis K, Kidd MR, Schattner P, et al. A bibliometric analysis of Australian general practice publications from 1980 to 2007 using PubMed. Inform Prim Care 2010;18:223-33

39. Mendis K, Solangaarachchi I. PubMed perspective of family medicine research: Where does it stand? Fam Pract 2005;22:570-5.

40. Steel N, Abdelhamid A, Stokes T, et al. A review of clinical practice guidelines found that they were often based on evidence of uncertain relevance to primary care patients. J Clin Epidemiol 2014;67:1251-7.

41. Amici JM, Rogues AM, Lasheras A, et al. A prospective study of the incidence of complications associated with dermatological surgery. Br J Dermatol 2005;153:967-71.
42. Futoryan T, Grande D. Postoperative wound infection rates in dermatologic surgery. Dermatol Surg 1995;21:509-14.

43. Lathlean S. Skin cancer in general practice in South Australia. A five year study Aust Fam Physician 1999;28(Suppl 1):S28-31.

44. Bruce J, Russell EM, Mollison J, et al. The quality of measurement of surgica wound infection as the basis for monitoring: a systematic review. J Hosp Infect 2001;49:99-108.

45. Higgins JP, Altman DG, Gøtzsche PC, et al.; Cochrane Bias Methods Group; Cochrane Statistical Methods Group. The Cochrane Collaboration's tool for assessing risk of bias in randomised trials. BMJ 2011;343:d5928.

\section{Competing interests: None declared.}

This article has been peer reviewed.

Affiliations: Discipline of General Practice and Rural Medicine (Charles, Heal, Delpachitra, Wohlfahrt, Hardy, Banks), Mackay Clinical School, College of Medicine and Dentistry, James Cook University, Mackay; Anton Breinl Research Centre for Health Systems Strengthening (Heal), Australian Institute of Tropical Health and Medicine, James Cook University, Townsville; Mackay Institute of Research and Innovation (Heal), Townsville; Paul Hopkins Medical Centre (Kimber, Sullivan), Mackay; Smart Scan Mackay (Browning), Mackay; Mareeba Medical Centre (Saednia), Mareeba; Tropical Health Solutions (Buttner), Townsville; Centre for Chronic Disease Prevention (Buttner), James Cook University, Cairns, Australia

Contributors: Daniel Charles and Clare Heal contributed equally to this manuscript and are joint first authors. Daniel Charles led development of the study protocol, imple- mentation of the trial at each practice, data cleaning and data analysis; he also assisted with data collection and data entry. Clare Heal conceived the study idea, orchestrated practice recruitment and data collection, and was the overseeing supervisor, with substantial input into all aspects of the project. Meth Delpachitra led the data-entry process and assisted Clare Heal and Daniel Charles in data collection. Michael Wohlfahrt, Debbie Kimber, Julie Sullivan, Sheldon Browning and Sabine Saednia led patient recruitment and data collection at their respective practices. Petra Buttner assisted with the sample size calculation and statistics. Alexandra Hardy assisted with the development of the study design and protocol, and with data cleaning and checking. Jennifer Banks assisted with development of the study design and protocol. All authors contributed to drafting and revising the manuscript for important intellectual content, approved the final version for publication and agreed to act as guarantors of the work.
Funding: This study received funding from the Royal Australian College of General Practitioners P.W.H. Grieve Award, a Royal Australian College of General Practitioners Family Medical Care Education and Research Grant, the Mackay Private Practitioners Fund and a James Cook University Honours Program grant.

Data sharing: All portions of the data are available for sharing, upon contact with the corresponding author.

Acknowledgements: The authors thank Debbie Skerman, Lynn Wohlfahrt, Robyn Cavanagh, Emma Nicholas Pugh, Niky Cek, Dr. Luke Notley, Dr. Andrew O'Neil, Dr. Andrea Cosgrove, Dr. Grant Manypenny, Dr. Martelle Botha and Jaclyn Louise Atkinson, as well as all doctors and nurses at the Paul Hopkins Medical Centre, Smart Scan Mackay and Mareeba Medical Centre, for their assistance with data collection.

Accepted: Mar. 15, 2017

Correspondence to: Clare Heal, Clare.heal@ jcu.edu.au 